\title{
THE EOQ MODEL: A DIFFERENTIAL CYCLIC SYSTEM FOR CALCULATING ECONOMIC ORDER QUANTITY
}

\author{
RABHA W. IBRAHIM ${ }^{1, *}$, SAMIR B. HADID ${ }^{2}$ \\ ${ }^{1}$ Faculty of Computer Science and Information Technology, University Malaya, 50603, Malaysia \\ ${ }^{2}$ Ajman University of Science and Technology, College of Education and Basic Sciences, UAE \\ *Corresponding author: rabhaibrahim@yahoo.com
}

\begin{abstract}
The EOQ model determines the quantity that minimizes the total sum of all cost functions. We suggest a common structure for economic order quantity type non-linear differential models with costs functions with respect to time in a cyclic period. For this model, we analyze the related optimization problem and develop a relaxed method for determining a bounded interval containing the optimal cycle length. Also for a special class of transportation functions, we study these consequences and introduce algorithms to calculate the optimal size and the corresponding optimal order stage.
\end{abstract}

\section{INTRODUCTION}

The general economy challenged by governments in recent years has been described by almost a linear model. For such a model, it must satisfy some kind of stability. Therefore, it would be problematic to consider it likely that decisions occupied on the basis of past actions could cause to accurate future consequences. In view of the uncertainty and complexity usual of this setting, the question arises of the need to seek out new methods to deal with it. One of the most dynamic features in managing any economic units is the Economic Order Quantity (EOQ). It might be summed up, among other features, as confirming both good customer service and efficient production while keeping records as low as possible.

Received 2018-01-27; accepted 2018-03-14; published 2018-05-02.

2010 Mathematics Subject Classification. 44A45.

Key words and phrases. differential system; economic order quantity; cost function.

(C) 2018 Authors retain the copyrights of their papers, and all open access articles are distributed under the terms of the Creative Commons Attribution License. 
The behavior of the cost function needs not be linear. Furthermore, to be able to cover all possible conditions, it would be sensible to signify them as variables with respect to time, position and controller. These models usually are driven analytically by suggesting bounded intervals containing an optimal cycle length (reorder interval) to arrive at concave functions. This method brings a minimal value of EOQ. For the other methods of cycle optimal lengths that evaluate the upper bound by an algorithm or by utilizing some concepts of the convex optimization problem. The convexity concept does not change in each bounded interval. In this case the procedure calculates the interval, such that the global minimum point resides. Another structure is that the cost function imposes in bounded intervals. Hence, the cost function is convex in all intervals; such an algorithm introduces a local solution in each interval (see [1]). Several generalizations and modifications can be suggested to the EOQ model, counting backordering costs and multiple units. Moreover, the economic order interval can be computed from the EOQ and the economic production quantity typical (which controls the optimal production quantity) can be calculated in a similar technique. The Baumol-Tobin model, has also been utilized to compute the money demand function, where a person's holdings of money balances can be realized in an approach parallel to a firm's holdings of inventory [2]. Malakooti presented a model with conditions that could be minimized the total cost, order quantity and Shortages [3]. A historical overview can be found in [4]. Recently, Lagodimoset et al. introduced a discrete-time EOQ model [5]. Methods for minimization can be found in [6].

In this paper, we introduce a new mathematical modeling for EOQ. Our method is based on the fixed point theorems in some of compact sets. The minimization of EOQ is described by considering a geometric frame for the total cost function during a time. Our study is related to the minimum norm solution. Examples of linear and non-linear systems illustrated in the sequel. Some of the systems have equilibrium points, which are given approximately. This technique allows EOQ problems to be under unfixed credit period, where the classical models are satisfied fixed credit period.

\section{Processing}

The model of EOQ was established by Ford W. Harris [7] under the formula:

$$
Q=\sqrt{2 A B / C}
$$

where $A$ is the annual demand quantity, $B$ is the fixed cost per item and $C$ is the annual holding cost per item. In this note we suggest that (2.1) changes over time taking the formula

$$
Q(\tau)=\sqrt{2 A(\tau) B(\tau) / C(\tau)}
$$


To minimize (2.2), we shall prepare a differential equation as follows: by squaring (2.2) and differentiating the result, we have

$$
\begin{aligned}
\frac{d}{d \tau} Q(\tau) & =\frac{Q(\tau)}{2}\left(\frac{A^{\prime}(\tau)}{A(\tau)}+\frac{B^{\prime}(\tau)}{B(\tau)}-\frac{C^{\prime}(\tau)}{C(\tau)}\right) \\
& :=Q(\tau)\left(f_{1}(\tau)+f_{2}(\tau)+f_{3}(\tau)\right) \\
& :=Q(\tau) F(\tau, A, B, C) .
\end{aligned}
$$

Eq. (2.3) is a general case of the suggested model (differential EOQ) given in [8]. Our aim is to find a minimum solution for (2.3). For this purpose, we introduce the following operator $\Pi: C \rightarrow C$, where $C$ is any closed bounded and convex subset of a Banach space $B$. The map $\Pi$ is called non-expensive if the following inequality holds:

$$
\|\Pi \xi-\Pi \eta\|<\|\xi-\eta\|
$$

and it is called a contraction mapping if

$$
\|\Pi \xi-\Pi \eta\| \leq \ell\|\xi-\eta\|, \quad \ell \in(0,1) .
$$

We need the following outcomes:

Lemma 2.1. ( Leray-Schauder theorem) Let $\Pi$ be a continuous and compact mapping of a Banach space $\mathbb{B}$ into itself, such that the set

$$
\{\xi \in \mathbb{B}: \xi=\lambda \Pi \xi \text { for some } 0 \leq \lambda \leq 1\}
$$

is bounded. Then $\Pi$ has a fixed point.

Lemma 2.2. (Zorn's Lemma) For any weakly compact convex subset $C$ and any non-expansive map $\Pi$ : $C \rightarrow C, C$ has a minimal (П -invariant) subset.

Lemma 2.3. [9] Let $\mathbb{B}$ be a strictly convex normed space with norm $\|$.$\| . The set of fixed points of a$ non-expansive mapping $\Pi: \mathbb{B} \rightarrow \mathbb{B}$ is either empty or closed and convex.

\section{Results}

In this section, we introduce two cases of the function $F$ in $(2.3)$.

Let the function $F, Q$ be continuous with respect to $\tau \in J:[0, T]$ and $F$ be Lipschitz with respect to $(A, B, C)$ then there is a positive constant $\wp \geq 0$ such that 


$$
\|F(\tau, A, B, C)-F(\tau, a, b, c)\| \leq \wp(\|A-a\|+\|B-b\|+\|C-c\|) .
$$

Moreover, we let $F(\tau, 0,0,0)=0$. Define the following operator:

$$
(\Pi)(Q)(\tau):=\int_{0}^{\tau} Q(\varsigma) F(\varsigma, A, B, C) d \varsigma, \quad Q(0)=0 .
$$

In our study, we involve some type of specification of how EOQ moves from step to step; that is $Q(\tau+1)=$ $\Pi Q(\tau)$. The stationary step can be defined as a case for which $\Pi Q(\tau)=Q(\tau)$. This can be viewed as an equilibrium point of the system. That is the fixed points of $\Pi$ are the states at which the process of EOQ is clear (stable).

We have the following result:

Theorem 3.1. Consider the Eq (2.3). If

$$
\wp T(\|A\|+\|B\|+\|C\|) \geq 1
$$

then it admits at least one solution. If

$$
\wp T(\|A\|+\|B\|+\|C\|)<1
$$

then it admits a unique solution which minimizes the problem (2.2).

Proof. Obviously,

$$
\begin{aligned}
|(\Pi)(Q)(\tau)| & =\left|\int_{0}^{\tau} Q(\varsigma) F(\varsigma, A, B, C) d \varsigma\right| \\
& \leq \int_{0}^{\tau}|Q(\varsigma)||F(\varsigma, A, B, C)| d \varsigma \\
& =\int_{0}^{\tau}|Q(\varsigma) \| F(\varsigma, A, B, C)-F(\tau, 0,0,0)| d \varsigma \\
& \leq \wp T(\|A\|+\|B\|+\|C\|)\|Q\| ;
\end{aligned}
$$

consequently, we obtain

$$
\frac{1}{\wp T(\|A\|+\|B\|+\|C\|)}|(\Pi)(Q)(\tau)|:=\lambda|(\Pi)(Q)(\tau)| \leq\|Q\| .
$$

By taking the maximum value, we get

$$
\lambda\|(\Pi)(Q)\|=\|Q\|
$$


The operator $\Pi$ is continuous, bounded and compact in a subset of Banach space $\mathbb{B}$. Hence, in view of Lemma 2.1, the (2.3) has a solution. Let $P \in \mathbb{B}$ such that

$$
(\Pi)(P)(\tau)=\int_{0}^{\tau} P(\varsigma) F(\varsigma, A, B, C) d \varsigma, \quad P(0)=0
$$

A calculation implies that

$$
\begin{aligned}
|(\Pi)(Q)(\tau)-(\Pi)(P)(\tau)| & =\left|\int_{0}^{\tau} Q(\varsigma) F(\varsigma, A, B, C) d \varsigma-\int_{0}^{\tau} P(\varsigma) F(\varsigma, A, B, C) d \varsigma\right| \\
& \leq \int_{0}^{\tau}|Q(\varsigma)-P(\varsigma)||F(\varsigma, A, B, C)| d \varsigma \\
& \leq T|F(\varsigma, A, B, C)-F(\varsigma, 0,0,0)|\|Q-P\| \\
& \leq \wp T(\|A\|+\|B\|+\|C\|)\|Q-P\| ;
\end{aligned}
$$

If $\wp T(\|A\|+\|B\|+\|C\|)<1$ then by Banach fixed point theorem, we conclude that (2.3) has a unique solution. It is clear that

$$
\|(\Pi)(Q)-(\Pi)(P)\| \leq\|Q-P\| .
$$

Thus operator $\Pi$ is not-expensive map. Therefore, in view of Lemma 2.2 and Lemma 2.3, the set of fixed point is a minimal close convex set. Note that the fixed point corresponds to equilibrium of EOQ.

\section{EXAMPLES}

In this section, we shall consider some applications.

Example 1. Assume that a company's production in two seasons during a year satisfying the following EOQ system:

$$
\begin{aligned}
& Q(1)=A(1) B(1)-C(1) Q(2) \\
& Q(2)=A(2) B(2)-C(2) Q(1) .
\end{aligned}
$$

Define the company's reaction function $\rho: \mathbb{R}_{+}^{2} \rightarrow \mathbb{R}_{+}^{2}$ by

$$
\rho(Q(1), Q(2)):=(A(1) B(1)-C(1) Q(2), A(2) B(2)-C(2) Q(1))
$$


If $\max \{C(1), C(2)\}<1$ then a computation with respect to city block metric, gives

$$
\begin{aligned}
\left|\rho(\mathbf{Q})-\rho\left(\mathbf{Q}^{\prime}\right)\right| & =\left|A(1) B(1)-C(1) Q(2)-\left(A(1) B(1)-C(1) Q^{\prime}(2)\right)\right| \\
& +\left|A(2) B(2)-C(2) Q(1)-\left(A(2) B(2)-C(2) Q^{\prime}(1)\right)\right| \\
& =C(1)\left|Q^{\prime}(2)-Q(2)\right|+C(2)\left|Q^{\prime}(1)-Q(1)\right| \\
& \leq \max \{C(1), C(2)\}\left(\left|Q^{\prime}(2)-Q(2)\right|+\left|Q^{\prime}(1)-Q(1)\right|\right) \\
& =\max \{C(1), C(2)\}\left|\mathbf{Q}-\mathbf{Q}^{\prime}\right| .
\end{aligned}
$$

Thus, the existence and uniqueness outcome for the equilibrium, where $\rho$ has a unique fixed point $\mathbf{Q}^{*}$ if $C(i)<1, i=1,2$. We have the following facts:

- The condition $C(i)<1, i=1,2$ is sufficient for equilibrium but not necessary.

- This condition implies the convexity of the set of the fixed point ( Lemma 2.3).

- We may replace the metric with the Euclidean metric to obtain the same result.

- In view of the condition $C(i)<1, i=1,2$ the system (4.1) can be considered as a fuzzy system (see Fig. 1).

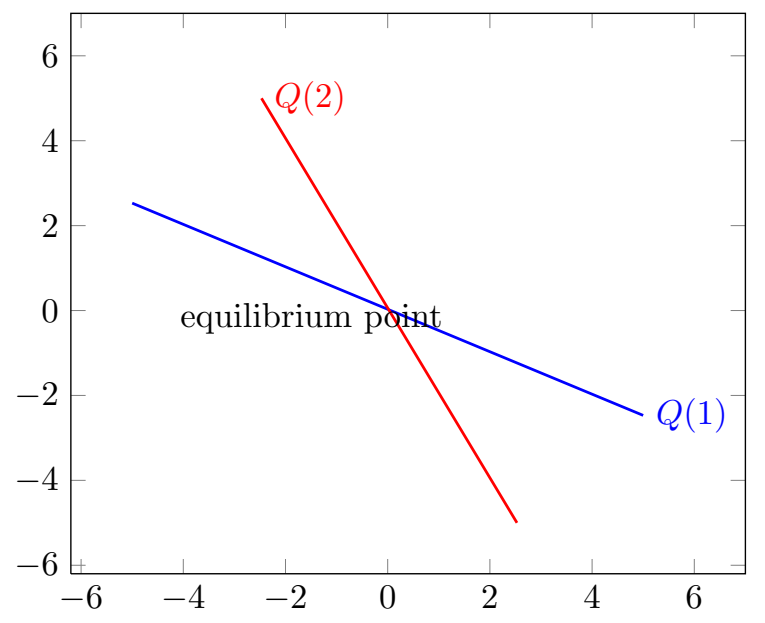

Figure 1: Example 1; the functions $Q(1)$ in the horizontal axis and $Q(2)$ in the vertical axis. Here, we suppose $C(i)=0.5, i=1,2$. and $A(i)=0.1, B(i)=0.3$.

Example 2. Assume that a recent state of market is $Q(0)=\left(Q_{1}(0), Q_{2}(0)\right)$. A company $j$ is imposing $Q_{j}(0)$ units. At time $\tau=1$ each company selects its outcome level $Q_{j}(1)$ by replaying to the quantity. Consequently, we have the system (see Fig.2)

$$
\begin{aligned}
& Q_{1}(\tau+1)=A(1) B(1)-C(1) Q_{2}(\tau) \\
& Q_{2}(\tau+1)=A(2) B(2)-C(2) Q_{1}(\tau) .
\end{aligned}
$$


The sequence $<Q(\tau)>$ converges the equilibrium point $Q *$ whenever $\max \{C(1), C(2)\}<1$.

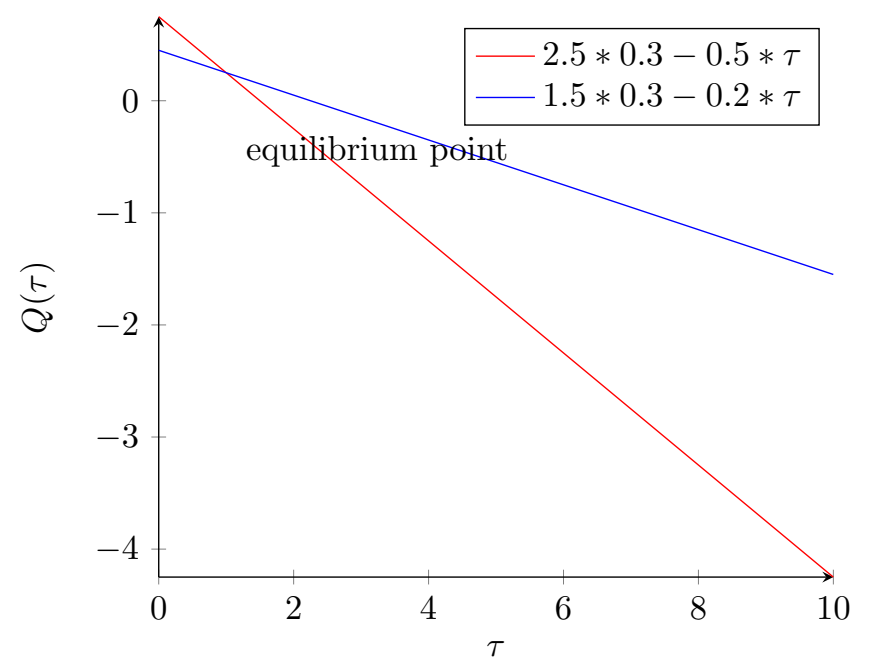

Figure 2: Example 2; the functions $Q(\tau)$ with respect to $\tau$. We suppose $C(1)=0.5, C(2)=0.2$.

Example 3. Suppose the following non-linear system

$$
\begin{aligned}
Q_{1}(\tau) & =\frac{1}{2\left(1+Q_{2}(\tau)\right)} \\
Q_{2}(\tau) & =\frac{\exp \left(-Q_{1}(\tau)\right)}{2} .
\end{aligned}
$$

It is clear that the function $\varrho: \mathbb{R}_{+}^{2} \rightarrow \mathbb{R}_{+}^{2}$, where

$$
\varrho\left(Q_{1}, Q_{2}\right)=\left(\frac{1}{2\left(1+Q_{2}(\tau)\right)}, \frac{\exp \left(-Q_{1}(\tau)\right)}{2}\right)
$$

is a contraction, so an equilibrium point exists and unique.

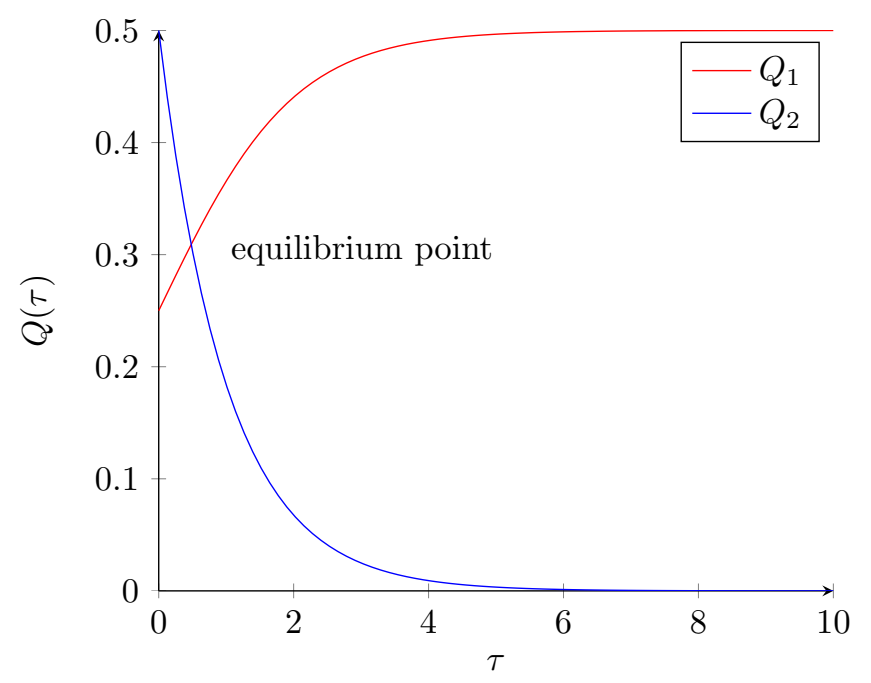

Figure 3: Example 3: the functions $Q(\tau)$ with respect to $\tau$. There is a unique equilibrium point for the system corresponding to a unique solution. 
Example 4. Suppose the following non-linear equation $Q: \mathbb{R}_{+} \rightarrow \mathbb{R}_{+}$

$$
Q(\tau)=\frac{1}{2}\left(\tau+C \frac{1}{\tau}\right), \quad \tau \neq 0 .
$$

It is clear that $\lim _{\tau \longrightarrow 0} Q(\tau)=\infty$. To minimize (2.2), we shall use the an approximation technique. The approximation form of (4.4) is as follows:

$$
\tau_{m+1}=\frac{1}{2}\left(\tau_{m}+C \frac{1}{\tau_{m}}\right)
$$

It is clear that this sequence converges to $\sqrt{C}$. Therefore, since $Q(\tau)$ is continuous, then $\sqrt{C}$ is a fixed point of $Q$. Also, in view of Theorem 3.1, for $0<\sqrt{C}<1$, Eq. (4.4) has a unique fixed point corresponding to the solution of it. The reaction of (4.5) showed that it has a limit and this limit converges to the fixed point of (4.4). Therefore, no need to show that the function $Q$ is a contraction mapping.

\section{Discussion}

In general, economic can be performed by a set of fixed points; thus fixed point theorems can provide the equilibria of economic. The frame of this work was to consider a new formula of EOQ Model. We introduced a technique of minimizing it by using the concept of fixed point theory. We developed this method to be suitable to the model (see Theorem 3.1). Applications are given including linear and non-linear systems. These systems are based on changing the solution during time in a fixed interval. The equilibrium points of each system was established, where it represented to the stationary state at which the markets clear. Also, this stationary state acted when the company wants to change its strategy.

\section{REFERENCES}

[1] R. W. Grubbstrom, Modelling production opportunities an historical overview, Int. J. Product. Econ. 41 (1995), 1-14.

[2] A Caplin, J. Leahy, John, Economic Theory and the World of Practice: A Celebration of the (S, s) Model, J. Econ. Persp. $24(1)(2010), 183-201$.

[3] B. Malakooti, Operations and Production Systems with Multiple Objectives (2013). John Wiley \& Sons.

[4] M.Holmbom, A. Segerstedt, Economic Order Quantities in production: From Harris to Economic Lot Scheduling Problems, Int. J. Product. Econ. 155 (2014), 82-90.

[5] A. G. Lagodimos, et al., The discrete-time EOQ model: Solution and implications, Eur. J. Oper. Res. 266 (2018) ,112-121.

[6] R. W. Ibrahim, Maximize minimum utility function of fractional cloud computing system based on search algorithm utilizing the Mittag-Leffler sum, Int. J. Anal. Appl. 16(1) (2018), 125-136.

[7] R. W. Harris, How Many Parts to Make at Once, Operat. Res. 38 (6)(1990), 947.

[8] G. Mahata, P Mahata, Analysis of a fuzzy economic order quantity model for deteriorating items under retailer partial trade credit financing in a supply chain, Math. Comput. Model. 53 (2011), 1621-1636.

[9] S.G. Ferreira, The existence and uniqueness of the minimum norm solution to certain linear and nonlinear problems, Signal Proc. 55 (1996), 137-139. 